\title{
Prevalencia de parásitos intestinales con riesgo zoonótico en Canis lupus familiaris de la localidad de Retes, Lima, Perú
}

\author{
Prevalence of intestinal parasites with zoonotic risk in Canis lupus \\ familiaris of Retes town, Lima, Peru
}

\author{
Asucena Naupay I. ${ }^{1}$, Julia Castro H. ${ }^{2}$, Manuel Tello A. ${ }^{1}$
}

\section{Resumen}

\begin{abstract}
El objetivo del presente estudio fue determinar la prevalencia de parásitos intestinales y los factores de riesgo asociados con la transmisión zoonótica en perros con dueño de la localidad rural de Retes, distrito de Huaral (Lima, Perú). Se realizó un estudio descriptivo de corte transversal, donde se recolectaron muestras fecales de 47 perros y fueron procesadas mediante los métodos directo simple, flotación de Willis-Molloy y sedimentación rápida modificada por Lumbreras. Así mismo, se hizo la identificación morfométrica de las especies parasitarias. El análisis estadístico se realizó mediante análisis bivariado con distribución Chi cuadrado. La prevalencia de enteroparasitosis fue de $31.9 \%$. Se halló Dipylidium caninum (12.8\%), Toxocara canis (10.6\%), Ancylostoma spp (4.3\%), Cystoisospora canis (4.3\%), Taenia spp (2.1\%). El monoparasitismo fue de $76.7 \%$, seguido de biparasitismo (13.4\%) entre helmintos y protozoarios. Se halló como único factor de riesgo asociado al parasitismo intestinal el lugar de alimentación $(\mathrm{OR}=7.11$; $\mathrm{X}^{2}=5.23 ; \mathrm{p}=0.03$ ). Los helmintos zoonóticos del perro D. caninum, T. canis y Ancylostoma spp, dada la prevalencia encontrada, podrían transmitirse fácilmente al ser humano pues se encuentran ocupando el mismo hábitat en la zona rural de Huaral.
\end{abstract}

Palabras clave: $\quad$ zoonosis; Toxocara canis; Dipylidium caninum; helmintos; coccidios; Ancylostomideos

\footnotetext{
${ }^{1}$ Laboratorio de Parasitología Humana y Animal, Facultad de Ciencias Biológicas, Universidad Nacional Mayor de San Marcos, Lima, Perú

${ }^{2}$ Laboratorio de Control de Artrópodos y Vectores, Facultad de Ciencias Biológicas, Universidad Nacional Mayor de San Marcos, Lima, Perú

${ }^{3}$ E-mail: asucnau@yahoo.es
} 
The aim of this study was to determine the prevalence of intestinal parasites and the risk factors associated with zoonotic transmission in dogs with owner in the rural town of Retes, Huaral district (Lima, Peru). A descriptive cross-sectional study was carried out, where fecal samples of 47 dogs were collected and processed by simple direct methods, Willis-Molloy flotation technique and the rapid sedimentation technique modified by Lumbreras. The morphometric identification of the parasitic species was also done. The statistical analysis was performed by bivariate analysis with Chi square distribution. The prevalence of enteroparasitosis was $31.9 \%$. Dipylidium caninum $(12.8 \%)$, Toxocara canis (10.6\%), Ancylostoma spp (4.3\%), Cystoisospora canis (4.3\%), Taenia spp (2.1\%) were found. Monoparasitism was $76.7 \%$, followed by biparasitism (13.4\%) between helminths and protozoa. The feeding site was the only risk factor associated with intestinal parasitism $\left(\mathrm{OR}=7.11, \mathrm{X}^{2}=5.23, \mathrm{p}=0.03\right)$. Dog's zoonotic helminths D. caninum, T. canis and Ancylostoma spp, given the prevalence found, could easily be transmitted to humans because they are occupying the same habitat in the rural area of Huaral.

Key words: zoonoses; Toxocara canis; Dipylidium caninum; helminths; coccidia; Ancylostomideos

\section{INTRODUCCIÓN}

Las poblaciones suburbanas y rurales del Perú carecen de condiciones sanitarias básicas, producto de la pobreza y del desconocimiento elemental sobre la crianza y cuidados de los animales domésticos, especialmente de animales que conviven con las familias. El caso del perro como animal de compañía, que en la vivienda rural actúa además como guardián, constituye un factor de riesgo en la transmisión de infecciones parasitarias al ser humano por el contacto continuo con sus dueños.

Los perros albergan en su sistema digestivo a protozoarios, nematodos $\mathrm{y}$ platelmintos (Hendrix, 1999; Leguía, 2002, Quiroz, 2008). Los helmintos intestinales que afectan frecuentemente a los perros son Ancylostoma caninum, Trichuris vulpis, Strongyloides sp, Dipylidium caninum y Toxocara canis (Giraldo et al., 2005; Huerto et al., 2015; Minaya, 2016), los cuales ocasionan el deterioro de la salud del animal y, en algunos casos, le ocasionan la muerte.
Algunas infecciones parasitarias transmitidas por los perros son causantes de zoonosis, especialmente afectando a la población infantil de condición económica baja con hábitos y condiciones higiénico-sanitarias deficientes. Entre las helmintiasis se encuentra la toxocariosis humana, que se produce por la ingesta accidental de huevos infectivos de $T$. canis, y que causa los síndromes de larva migrante ocular (LMO) y larva migrante visceral (LMV) (Glickman y Schanstz, 1981; Acha y Szyfres, 2003; Rivarola et al., 2009; Quercia et al., 2015). La segunda zoonosis de importancia se debe a la penetración y desplazamiento a través de la piel de larvas de Ancylostoma brazilensis, Uncinaria stenocephala y Ancylostoma caninum, que ocasionan el síndrome de larva migrante cutánea (LMC) (Heukelbach y Feldmeier, 2008; Galarza et al., 2009; Feldmeier y Schuster, 2012) y la infección por Strongyloides de origen canino (Gorman et al., 2006). Otra helmintiasis de importancia zoonótica es la dipilidiasis ocasionada por la ingesta accidental de pulgas parasitadas con larvas cisticercoides de Dipylidium caninum (Molina et al., 2003). 
Las investigaciones sobre prevalencia de enteroparásitos en perros con dueño realizadas en Latinoamérica reportan resultados variables. Así, los trabajos realizados en Chile indican prevalencias entre 4.5 y $78 \%$ (Sandoval, 2003; Gorman et al., 2006), en Brasil entre 0.7 y $23.6 \%$ (Oliveira-Sequeira et al., 2002), en Argentina entre 5 y $41 \%$ (Torno et al., 1996; Betti et al., 2007; Camaño et al., 2010), en México entre 0.7 a 37\% (Rodríguez et al., 2001; Martínez-Barbabosa et al., 2015), en Venezuela entre 76.5 y $88.8 \%$ (Tortolero et al., 2008; Cazorla y Morales, 2013) y en Colombia entre 37.4 y $76 \%$ (Vásquez et al., 2004; Giraldo et al., 2005; Alarcón et al., 2015; Gonzales y Giraldo, 2015).

Los estudios realizados en el Perú en perros con dueño de zonas rurales son pocos. Entre ellos, los efectuados por Huerto et al. (2015) en Huánuco con una prevalencia de $92.3 \%$, de Vilca y Anccasi (2013) en Puno con $78.6 \%$ y el de Minaya (2016) en Junín quién reportó $73.2 \%$. También se realizaron estudios en zonas urbanas de Lima por Caro et al. (2013) y Naupay et al. (2017) encontrando prevalencias de 18 y $48.6 \%$ en cada caso, y en Ica por Trillo-Altamirano et al. (2003), quienes reportaron una prevalencia de $40.1 \%$.

El presente estudio tuvo como objetivo determinar la prevalencia de parásitos intestinales zoonóticos en perros con dueño e identificar factores de riesgo asociados en el distrito de Huaral, departamento de Lima, para el establecimiento de medidas de prevención y control.

\section{Materiales y Métodos}

\section{Lugar de Estudio}

Se realizó un estudio descriptivo de corte transversal en el centro poblado de Retes, que pertenece al distrito de Huaral, provincia de Lima (Perú). Retes tiene una población de 100434 habitantes (INEI, 2015), un área territorial de $640.76 \mathrm{~km}^{2}$, se encuentra a 182 msnm y presenta un clima seco y semicálido. La temperatura media anual es de $19.4{ }^{\circ} \mathrm{C}$.

\section{Población Canina}

La población de estudio estuvo conformada por 47 ejemplares de perros domésti$\cos$ (Canis lupus familiaris) que conviven con sus dueños. Se tomó como factor de inclusión a todos los perros que recibieron tratamiento antiparasitario no menor a 15 días antes de la toma de muestra y que sus dueños dieron el consentimiento. El tamaño de la muestra se obtuvo mediante la fórmula para una población infinita o desconocida, según Spiegel y Stephens (2009). El nivel de confianza fue del $95 \%$ y el error previsto del $14.29 \%$. La visita domiciliaria se coordinó con el dueño de la mascota, los datos recopilados fueron anotados en una ficha epidemiológica en la que se consideraron las variables edad, sexo, raza, convivencia con otros animales, tratamiento antiparasitario, sintomatología, tipo de alimentación, lugar de alimentación y lugar de defecación.

\section{Toma de Muestra}

Las muestras de heces fueron recolectadas en dos días consecutivos. El primer día se hizo entrega de kits que contenían una bolsa ziploc con un frasco rotulado y un palito bajalengua para la toma de la muestra, y en el segundo día se recogieron las muestras fecales de los domicilios participantes. Las 47 muestras de heces se preservaron con formol-sal al 5\% y fueron llevadas al Laboratorio de Parasitología Humana y Animal de la Facultad de Ciencias Biológicas de la Universidad Nacional Mayor de San Marcos, Lima, para el análisis coproparasitológico.

\section{Diagnostico Coproparasitológico}

Las muestras se procesaron por los métodos coproparasitoscópicos directo simple, flotación de Willis-Molloy y sedimentación rápida modificada por Lumbreras et al. 
Cuadro 1. Prevalencia de parásitos intestinales en 47 perros con dueño de la localidad de Retes, Huaral, Lima (Perú)

\begin{tabular}{lcc}
\hline Especie & N. & $\begin{array}{c}\text { Prevalencia } \\
(\%)\end{array}$ \\
\hline Cestodos & & \\
$\quad$ Dipylidium caninum & 6 & 12.8 \\
$\quad$ Taenia spp & 1 & 2.1 \\
Nematodos & & \\
$\quad$ Toxocara canis & 5 & 10.6 \\
$\quad$ Ancylostoma spp & 2 & 4.3 \\
Protozoarios & & \\
$\quad$ Cystoisospora canis & 2 & 4.3 \\
\hline
\end{tabular}

Cuadro 2. Frecuencia de asociaciones parasitarias en perros de la localidad de Retes, Huaral, Lima (Perú)

\begin{tabular}{lcc}
\hline Asociación & $\mathrm{N}^{\circ}$ & $\%$ \\
\hline Monoparasitismo & & \\
$\quad$ Dipylidium caninum & 5 & 33.3 \\
Toxocara canis & 3 & 20.0 \\
Ancylostoma spp & 2 & 16.7 \\
$\quad$ Taenia spp & 1 & 6.7 \\
Biparasitismo & & \\
T. canis + Cystoisospora & 1 & 6.7 \\
spp & & \\
D. caninum + & 1 & 6.7 \\
Cystoisospora spp & & \\
\hline
\end{tabular}

(1962). Fueron observadas por duplicado en un microscopio de contraste de fase Karl Zeiss. La identificación se realizó a través de las características morfométricas de los parásitos (Rojas, 2003; Quiroz, 2009).

\section{Análisis Estadístico}

Para el análisis estadístico se usaron las tablas de contingencia de $2 \times 2$ para el cálculo del Odds ratio (OR) con un índice de con- fianza (IC) de1 95\%, Chi cuadrado de Pearson, y la razón de verosimilitud para cada una de las variables. El software usado fue SPSS v. 24.

\section{Resultados}

En el 31.9\% (15/47) de las muestras fecales se encontró una o más especies parasitarias entre helmintos y/o protozoarios. $\mathrm{La}$ mayor prevalencia de helmintos intestinales fue de tipo zoonótico como Dipylidium caninum (12.8\%), Toxocara canis (10.6\%) y Ancylostoma spp (4.3\%). También se encontraron huevos de otros helmintos que no son parásitos del perro como Hymenolepis diminuta y Ascaris spp, así como una baja prevalencia de protozoarios. La distribución porcentual de las especies parásitas se presenta en el Cuadro 1.

La asociación parasitaria más frecuente fue el monoparasitismo (76.7\%) con predominio de Dipylidium caninum (33.3\%) seguido del biparasitismo (13.4\%) entre helmintos y protozoarios (Cuadro 2).

Los resultados de análisis de las variables raza, sexo, edad, convivencia con otros animales, tratamiento antiparasitario, síntomas, tipo de alimentación, lugar de alimentación y lugar de defecación, relacionados con la frecuencia de perros con dueño en la localidad del estudio, mostró que la mayor población de perros fue de raza mestiza o cruzada $(59.6 \%)$ y el resto de raza definida (40.4\%). El 55.3\% fueron machos. Los perros, al estratificarse por grupo etario, correspondieron al $55.3 \%$ entre 0 y 1 año y el $44.7 \%$ a más de un año. La convivencia con otros animales como cuyes, conejos, gallinas y cerdos mostró una mayor frecuencia $(70.2 \%)$ que de aquellos que no convivían con otras especies (29.8\%) (Cuadro 3).

Los dueños de los perros informaron que el $44.7 \%$ recibió tratamiento antiparasitario reciente y el 55.3\% restante no recibió tratamiento antiparasitario, mientras que el 8.5\% 
Cuadro 3. Variables relacionadas con la frecuencia de perros con dueño de la localidad de Retes, Huaral, Lima (Perú)

\begin{tabular}{llcc}
\hline \multirow{2}{*}{ Factores } & & \multicolumn{2}{c}{ Frecuencia } \\
\cline { 3 - 4 } & & $\mathrm{n}$ & $\%$ \\
\hline Raza & Mestiza & 28 & 59.6 \\
\multirow{2}{*}{ Sexo } & Puro & 19 & 40.4 \\
\multirow{2}{*}{ Edad (en años) } & Macho & 26 & 55.3 \\
\multirow{2}{*}{ Convivencia con otros animales } & Hembra & 21 & 44.7 \\
\multirow{2}{*}{ Tratamiento antiparasitario } & $0-1$ & 26 & 55.3 \\
\multirow{2}{*}{ Signos clínicos } & $>1$ & 21 & 44.7 \\
\multirow{2}{*}{ Tipo de alimentación } & Sí & 33 & 70.2 \\
& No & 14 & 29.8 \\
Lugar de alimentación & Sí & 21 & 44.7 \\
\multirow{2}{*}{ Lugar de defecación } & No & 26 & 55.3 \\
& Sí & 4 & 8.5 \\
& No & 43 & 91.5 \\
& De casa & 40 & 85.1 \\
& Procesada & 7 & 14.9 \\
& En casa & 41 & 87.2 \\
& Calle & 6 & 12.8 \\
& Casa & 39 & 83.0 \\
& Calle & 8 & 17.0 \\
\hline
\end{tabular}

de los perros presentó algún signo clínico específico como vómitos, diarreas y falta de apetito. La mayoría de los perros se alimenta en el patio o techo de la vivienda $(87.2 \%)$ y depositan sus excretas en dichos lugares (83\%).

En los Cuadros 4 y 5 se presenta el nivel de asociación de las variables en estudio con la prevalencia de parásitos intestinales. En los perros mestizos se halló el mayor porcentaje de infección (39.3\%), principalmente con helmintos zoonóticos (Toxocara canis ([14.3\%], Dypilidium caninum [14.3\%] y Ancylostoma sp [7.1\%]), pero no se halló asociación significativa con respecto al sexo. Los perros menores de un año fueron los más parasitados (38.5\%) con helmintos zoonóticos, aunque sin asociación significativa. En for- ma similar, perros con convivencia con otros animales estuvieron más parasitados (33.3\%), pero sin asociación significativa. Así mismo, los perros que no recibieron tratamiento antiparasitario (34.6\%) y los que no presentaron signos clínicos $(27.7 \%)$ estuvieron más infectados con helmintos zoonóticos, pero sin que hubiera asociación significativa.

La única variable asociada a la prevalencia de parásitos intestinales fue el lugar de alimentación, donde el $50 \%$ de los perros que se alimentan en la calle estaban infectados con algún helminto con riesgo zoonótico $\left(\mathrm{OR}=7.11 ; \mathrm{X}^{2}=5.23 ; \mathrm{p}=0.03\right)$, mientras que los que se alimentan en la casa alcanzó el $28.2 \%$ de prevalencia de parásitos intestinales y positivos para helmintos zoonóticos. 
Cuadro 4. Asociación entre las variables de estudio y la prevalencia de parásitos intestinales en 47 perros con dueño de la localidad de Retes, Huaral, Lima (Perú) mediante el Chi cuadrado de Pearson

\begin{tabular}{|c|c|c|c|c|c|c|c|c|}
\hline \multirow{2}{*}{ Factores } & & \multirow{2}{*}{$\begin{array}{c}\text { Infectado } \\
(\%)\end{array}$} & \multicolumn{2}{|c|}{ Factor de riesgo } & \multicolumn{4}{|c|}{ Chi cuadrado } \\
\hline & & & OR & IC (95\%) & $\mathrm{X}^{2}$ & $\mathrm{p}$ & $\mathrm{RV}$ & $\mathrm{p}$ \\
\hline Raza & $\begin{array}{l}\text { Mestiza } \\
\text { Puro }\end{array}$ & $\begin{array}{l}39.3 \\
21.1\end{array}$ & 2.96 & $0.69-12.7$ & 2.25 & 0.13 & 2.36 & 0.12 \\
\hline Sexo & $\begin{array}{l}\text { Macho } \\
\text { Hembra }\end{array}$ & $\begin{array}{l}34.6 \\
28.6\end{array}$ & 1.09 & $0.30-3.92$ & 0.02 & 0.9 & 0.02 & 0.9 \\
\hline Edad & $\begin{array}{l}<1 \text { año } \\
>1 \text { año }\end{array}$ & $\begin{array}{l}38.5 \\
14.3\end{array}$ & 0.27 & $0.62-1.14$ & 3.39 & 0.07 & 3.56 & 0.06 \\
\hline $\begin{array}{l}\text { Convivencia con } \\
\text { otros animales }\end{array}$ & $\begin{array}{l}\mathrm{Si} \\
\mathrm{No}\end{array}$ & $\begin{array}{l}33.3 \\
28.6\end{array}$ & 1.07 & $0.27-4.28$ & 0.00 & 0.93 & 0.00 & 0.93 \\
\hline $\begin{array}{l}\text { Tratamiento } \\
\text { antiparasitario }\end{array}$ & $\begin{array}{l}\mathrm{Si} \\
\text { No }\end{array}$ & $\begin{array}{l}28.6 \\
34.6\end{array}$ & 1.42 & $0.38-5.24$ & 0.28 & 0.6 & 0.28 & 0.59 \\
\hline Signos clínicos & $\begin{array}{l}\mathrm{Si} \\
\mathrm{No}\end{array}$ & $\begin{array}{l}50.0 \\
30.2\end{array}$ & 0.69 & $0.57-0.85$ & 1.67 & 0.19 & 2.73 & 0.09 \\
\hline $\begin{array}{l}\text { Tipo de } \\
\text { alimentación }\end{array}$ & $\begin{array}{l}\text { De casa } \\
\text { Procesada }\end{array}$ & $\begin{array}{l}22.5 \\
85.7\end{array}$ & 0.95 & $0.16-5.63$ & 0.00 & 0.95 & 0.00 & 0.95 \\
\hline $\begin{array}{l}\text { Lugar de } \\
\text { alimentación }\end{array}$ & $\begin{array}{l}\text { En casa } \\
\text { Calle }\end{array}$ & $\begin{array}{l}29.3 \\
50.0\end{array}$ & 7.11 & $1.12-45.29$ & 5.23 & 0.02 & 4.64 & 0.03 \\
\hline $\begin{array}{l}\text { Lugar de } \\
\text { defecación }\end{array}$ & $\begin{array}{l}\text { Casa } \\
\text { Calle }\end{array}$ & $\begin{array}{l}28.2 \\
50.0\end{array}$ & 3.33 & $0.69-16.07$ & 2.41 & 0.12 & 2.21 & 0.14 \\
\hline
\end{tabular}

$\mathrm{OR}=$ odds ratio; $\mathrm{IC}=$ =intervalo de confianza; $\mathrm{X}^{2}=$ Chi cuadrado de Pearson; $\mathrm{p}=$ significancia; $\mathrm{RV}=$ razón de verosimilitud

\section{Discusión}

La prevalencia de parásitos intestinales en perros con dueño de la localidad de Retes fue de $31.9 \%$, concordando con otros reportes latinoamericanos (Gorman et al., 2006; Betti et al., 2007; Martínez-Barbabosa et al., 2015). Así mismo, estos resultados son comparables al $20.5 \%$ de helmintiasis en perros pastores en zonas rurales de Lampa y Carabaya, Puno (Cruz et al., 2012). No obstante, en otros estudios de perros con dueño se han reportado mayores prevalencias de parasitismo intestinal canino; así Vilca y Anccasi (2013) encontraron una prevalencia de $78.6 \%$ en la provincia de Puno, Huerto et al. (2015) de $92.3 \%$ en Huánuco y, recientemente, Minaya (2016) de $73.2 \%$ en el distrito de Canchayllo, Junín. Las diferencias entre estudios pueden deberse a factores climáticos (temperatura, humedad) y tipos de suelo. Asimismo, otro factor ambiental de importancia epidemiológica en la transmisión de enteroparásitos a los perros son las condiciones higiénico-sanitarias de cada centro poblado.

En el estudio se evidenció cinco especies de parásitos, comparables con otros estudios similares realizados en otras zonas rurales del Perú (Huerto et al., 2015; Minaya, 2016) con excepción del estudio de Cruz et al. (2012) en Puno, que registraron nueve especies, entre protozoarios y helmintos.

Dipylidium caninum fue el parásito zoonótico de mayor prevalencia $(12.8 \%)$, resultado que concuerda con la prevalencia encontrada por Huerto et al. (2015) en 
Cuadro 5. Asociación entre los factores de riesgo y la prevalencia de parasitismo por helmintos zoonóticos en perros con dueño de la localidad de Retes, Huaral, Lima (Perú)

\begin{tabular}{|c|c|c|c|c|c|}
\hline Factores & & $\begin{array}{l}\text { Toxocara } \\
\text { canis }\end{array}$ & $\begin{array}{c}\text { Ancylostoma } \\
\text { spp }\end{array}$ & $\begin{array}{l}\text { Dypilidium } \\
\text { caninum }\end{array}$ & $\mathrm{p}$ \\
\hline \multirow[t]{2}{*}{ Raza } & Mestiza & 4 & 2 & 4 & \multirow[t]{2}{*}{0.12} \\
\hline & Puro & 1 & -- & 2 & \\
\hline \multirow[t]{2}{*}{ Sexo } & Macho & 2 & 2 & 3 & \multirow[t]{2}{*}{0.9} \\
\hline & Hembra & 3 & -- & 3 & \\
\hline \multirow[t]{2}{*}{ Edad (años) } & $0-1$ & 5 & 2 & 3 & \multirow[t]{2}{*}{0.06} \\
\hline & $>1$ & -- & -- & 3 & \\
\hline \multirow{2}{*}{$\begin{array}{l}\text { Convivencia con otros } \\
\text { animales }\end{array}$} & Sí & 3 & -- & 6 & \multirow[t]{2}{*}{0.93} \\
\hline & No & 2 & 2 & -- & \\
\hline \multirow{2}{*}{$\begin{array}{l}\text { Tratamiento } \\
\text { antiparasitario }\end{array}$} & Sí & 2 & -- & 3 & \multirow[t]{2}{*}{0.59} \\
\hline & No & 3 & 2 & 3 & \\
\hline \multirow[t]{2}{*}{ Síntomas } & Sí & -- & -- & -- & \multirow[t]{2}{*}{0.09} \\
\hline & No & 5 & 2 & 6 & \\
\hline \multirow[t]{2}{*}{ Tipo de alimentación } & De casa & 5 & 2 & 4 & \multirow[t]{2}{*}{0.95} \\
\hline & Procesada & -- & -- & 2 & \\
\hline \multirow{2}{*}{ Lugar de alimentación } & En casa & 3 & 1 & 5 & \multirow[t]{2}{*}{0.03} \\
\hline & Calle & 2 & 1 & 1 & \\
\hline \multirow[t]{2}{*}{ Lugar de defecación } & Casa & 2 & 1 & 5 & \multirow[t]{2}{*}{0.14} \\
\hline & Calle & 3 & 1 & 1 & \\
\hline
\end{tabular}

Huánuco, Perú de $13.5 \%$, con Camaño et al. (2010) en Argentina de 12.6\%, y de Sandoval (2003) en Chile de 10\%. Su ciclo de vida está relacionado con la presencia de pulgas infectadas con cisticercoides, donde la ingesta accidental de estos insectos parasitados por las personas provocaría la dipilidiasis humana (Devera y Campos, 1998). Sin embargo, no se conoce la magnitud de esta zoonosis en el país, a pesar del proceso de ruralización de las ciudades como producto de las migraciones, que hace posible la crianza de las mascotas en el interior de las viviendas.

El segundo parásito de importancia zoonótica encontrado en el estudio fue Toxocara canis, parásito de amplia distribución mundial. La prevalencia de $10.6 \%$ fue similar a la prevalencia del $14 \%$ hallada en
México por Martínez-Barbabosa et al. (2011), de $9.1 \%$ en Santiago de Chile (Gorman et al., 2006) y de $8.6 \%$ en Coyaima, Colombia (Gonzales y Giraldo, 2015), pero menor al de $31.8 \%$ reportado en Falcón, Venezuela (Tortolero et al., 2008). En el Perú se han encontrado prevalencias mayores; así, de $54.8 \%$ en Huánuco (Huerto et al., 2015), de $41.5 \%$ en Canchayllo, Junín (Minaya, 2016) y de $49.3 \%$ en Puno (Vilca y Anccasi, 2013). Este problema zoonótico puede estar latente porque los perros callejeros contaminan profusamente el suelo con sus excretas.

La prevalencia de Ancylostoma spp fue baja (4.3\%); sin embargo, se debe tener en cuenta porque representa un riesgo para la salud humana y animal. En Huánuco, Huerto et al. (2015) hallaron el $72.1 \%$ de prevalen- 
cia, posiblemente debido a que es una región de clima tropical y subtropical que favorece su ciclo vital.

Entre los coccidios se encontró Cystoisospora canis (4.3\%), especie que comúnmente infecta a los perros (Tortolero et al., 2008). También se encontró Ascaris spp, H. diminuta y Taenia spp, posiblemente por la convivencia de los perros con otros animales y por las deficientes condiciones higiénico-sanitarias, lo que podría indicar que el perro está siendo un transmisor mecánico de estos parásitos.

La única asociación estadísticamente significativa fue entre el parasitismo intestinal con la variable «lugar de alimentación», posiblemente debido a que los perros que salen a la calle tienen mayor riesgo de adquirir una infección parasitaria. Por otro lado, se destaca la ausencia de signos clínicos intestinales en los perros a pesar de estar parasitados, constituyendo un factor de riesgo como diseminadores de los agentes parasitarios en la vivienda.

La ausencia de asociación de las otras variables con el parasitismo intestinal en este estudio concuerda con estudios similares realizados por otros investigadores (Vásquez et al., 2004; Alarcón et al., 2015), incluyendo el sexo (Gorman et al., 2006; Cazorla y Morales, 2013; Gonzales y Giraldo, 2015), mientras que Martínez-Barbabosa et al. (2011) encontraron asociación significativa con el tratamiento parasitario.

No se halló asociación significativa entre la edad y los helmintos zoonóticos pese a que los perros menores de un año estaban más parasitados con los helmintos zoonóticos, a diferencia de otros autores que encontraron que la edad y este tipo de helmintos estaban altamente asociados (Trillo et al., 2003; Llanos et al., 2010; Gonzales y Giraldo, 2015). La mayor prevalencia en perros mestizos ha sido igualmente encontrada por otros autores (Tortolero et al., 2008; Alarcón et al., 2015).

\section{Conclusiones}

- Se registra la prevalencia de parásitos intestinales con riesgo zoonótico en perros con dueño en la zona rural de Retes, Huaral (Lima).

- Los helmintos intestinales zoonóticos de mayor prevalencia fueron Dipylidium caninum, Toxocara canis y Ancylostoma spp.

- El lugar de alimentación fue encontrado como factor de riesgo asociado a la transmisión de parásitos intestinales de los perros con dueños en zona rural.

\section{Literatura Citada}

1. Acha PN, Szyfres B. 2003. Zoonosis y enfermedades transmisibles comunes al hombre y los animales. $3^{\text {a }}$ ed. Washington DC: OPS. 398 p.

2. Alarcón ZK, Juyo VY, Larrotta AJ. 2015. Caracterización epidemiológica de parásitos gastrointestinales zoonóticos en caninos con dueño del área urbana del Municipio de La Mesa, Cundinamarca. Rev Fac Med Vet Zootec 62: 20-36. doi: 10.15446/rfmvZ.v62n1.49382

3. Betti A, Cardillo N, Diez MI, Cornero F, Braida M, Agostini A. 2007. Parasitosis entéricas en caninos de un área del Gran Buenos Aires. 2003-2004. InVet 9: 53-58.

4. Camaño MC, López AE, Mozo G, Romero MS, Rivero AV, Saldaño $M B$, Soria EJ, et al. 2010. Parásitos intestinales de caninos y felinos. Prevalencia en barrios de la ciudad de Chumbicha. Ciencia 5: 57-69.

5. Caro J, Carrillo M, Hidalgo M, Fuentes I, Marthans A, Mateo C, Quispe C, et al. 2013. Parasitismo en el «perro peruano sin pelo» proveniente de hogares de Lima y Callao. En: XXII Reunión Científica del Instituto de Investigación de Ciencias Biológicas «Antonio Raimondi» UNMSM. Lima, Perú. 
6. Cazorla D, Morales P. 2013. Parásitos intestinales de importancia zoonótica en caninos domiciliarios de una población rural del estado Falcón, Venezuela. Bol Malariol Salud Amb 53: 19-28.

7. Cruz L, Chávez A, Falcón N, Fernández V, Huamán $H, L i O$, Huanca $W$. 2012. Helmintiasis en perros pastores de comunidades ganaderas de Puno, Perú. Rev Inv Vet Peru 23: 72-79. doi: 10.15381/rivep.v23i1.884

8. Devera J, Campos F. 1998. Dipilidiasis humana. Rev Biomed 9: 44-45.

9. Feldmeier H, Schuster A. 2012. Mini review: hookworm-related cutaneous larva migrans. Eur J Clin Microbiol 31: 915918. doi: 10.1007/s10096-011-1404-x

10. Galarza C, Ramos W, Guere P, Gutiérrez E, Tello M. 2009. Larva migrans cutánea simulando herpes zoster. Dermatol Peru 19: 58-61.

11. Giraldo MI, García NL, Castaño JC. 2005. Prevalencia de helmintos intestinales en caninos del departamento del Quindío, Colombia. Biomedica 25: 52346. doi: 10.7705/biomedica.v25i3.1359

12. Glickman LT, Schantz PM. 1981. Epidemiology and pathogenesis of zoonotic toxocariasis. Epidemiol Rev 3: 230-250. doi: 10.1093/oxfordjournals.epirev.a036235

13. Gonzalez AC, Giraldo JC. 2015. Prevalencia de parásitos intestinales zoonóticos en caninos (Canis lupus familiaris) del área urbana del municipio de Coyaima (Tolima). Rev Med 23: 24-34.

14. Gorman T, Soto A, Alcaino H. 2006. Parasitismo gastrointestinal en perros de comunas de Santiago de diferente nivel socioeconómico. Parasitol Latinoam 61: 126-132. doi: 10.4067/S0717-77122006000200005

15. Hendrix CM. 1999. Diagnostico parasitológico veterinario. $2^{\text {nd }} e d . M a-$ drid: Harcourt Brack. 326 p.

16. Heukelbach J, Feldmeier H. 2008. Epidemiological and clinical characteristics of hookworms-related cutaneous lar- va migrans. Lancet Infect Dis 8: 302-309. doi: 10.1016/S1473-3099(08)70098-7

17. Huerto E, Fonseca A, Dámaso B. 2015. Prevalencia de enteroparásitos zoonóticos en perros (Canis familiaris) y el nivel de cultura ambiental orientado a mascotas en Huánuco. Ágora 2: 233239. doi: 10.21679 /arc.v2i2.46

18. Leguía G. 2002. Enfermedades parasitarias de perros y gatos. Epidemiología y control. $2^{\circ}$ ed. Lima: Ed. Del Mar. 155 p.

19. Lumbreras H, Cantella R, Burga $R$. 1962. Acerca de un procedimiento de sedimentación rápida para investigar huevos de Fasciola hepatica en las heces, su evaluación y uso en el campo. Rev Méd Per 31: 167-174.

20. Llanos M, Condori M, Ibáñez T, LozaMurguía M. 2010. Parasitosis entérica en caninos (Canis familiaris) en el área urbana de Coroico, Nor Yungas Departamento de la Paz, Bolivia. J Selva Andina Res Soc 1: 37-49

21. Martínez-Barbabosa I, Gutiérrez M, Aguilar J, Pimienta R, Shea M. 2011. Frecuencia de geohelmintos en canes domiciliados en siete delegaciones de la Ciudad de México. Vet Méx 42: 83-91.

22. Martínez-Barbabosa I, Gutiérrez M, Ruiz LA, Fernández AM, Gutiérrez EM, Aguilar JM, Shea M, et al. 2015. Detección de Cryptosporidium spp y otros parásitos zoonóticos entéricos en perros domiciliados de la Ciudad de México. Arch Med Vet 47: 347-353. doi: 10.4067/S0301-732X2015000300012

23. Minaya AP. 2016. Identificación y frecuencia de parásitos gastrointestinales en canes de la SAIS Túpac Amaru en el distrito de Canchayllo, Jauja-Junín. Tesis de Médico Veterinario y Zootecnista. Lima, Perú: Univ. Peruana Cayetano Heredia. $24 \mathrm{p}$.

24. Molina CP, Ogburn J, Adegboyega P. 2003. Infection by Dipylidium caninum in an infant. Arch Pathol Lab Med 127: 157-159. doi: 10.1043/0003-9985(2003)127<e157:IBDCIA>2.0.CO;2 
25. Naupay A, Castro J, Cama M, Barboza K, Ayala R, Quispe G, Carbajal A, et al. 2017. Enteroparásitos zoonóticos de perros (Canis lupus familiaris) domiciliados en el AA HH Las Terrazas, Pamplona Alta, San Juan de Miraflores. En: XXVI Reunión Científica del Instituto de Investigación de Ciencias Biológicas «Antonio Raimondi» Univ. Nacional Mayor de San Marcos. Lima, Perú.

26. Oliveira-Sequeira TCG, Amarante AFT, Ferrari TB, Nunes LC. 2002. Prevalence of intestinal parasites in dogs from Sao Paulo State, Brazil. Vet Parasitol 103:19-27. doi: 10.1016/S03044017(01)00575-1

27. Quercia R, Sgroi M, Fandiño A, Costa M, Scovenna M, Parra A. 2015. Aspectos epidemiológicos, diagnósticos y de tratamiento de la toxocariosis ocular. Med Infantil 22: 98-105.

28. Quiroz H. 2009. Parasitología y enfermedades parasitarias de animales domésticos. México: Limusa. 876 p.

29. Rivarola M, Vuyk I, Riveros $M$, Canese A, Mico G. 2009. Toxocara canis en población pediátrica rural. Pediatría 36: 122-126.

30. Rodríguez-Vivas RI, Cob-Galera LA, Domínguez-Alpizar JL. 2001. Frecuencia de parásitos gastrointestinales en animales domésticos diagnosticados en Yucatán, México. Rev Biomed 12: 1925. doi: 10.32776/revbiomed.v12i1.253

31. Rojas M. 2003. Nosoparasitosis de perros y gatos peruanos. Lima, Perú: Martegraf. 83 p.
32. Sandoval BO. 2003. Determinación coproscópica de la fauna parasitológica en perros (Canis familiaris), en el área rural de Folilco, comuna de los Lagos, provincia de Valdivia, Décima Región, Chile. Tesis de Médico Veterinario. Valdivia, Chile: Univ. Austral de Chile. 44 p.

33. Spiegel M, Stephens L. 2009. Estadística Schaum. $4^{\mathrm{a}}$ ed. México DF: McGraw Hill. $577 \mathrm{p}$.

34. Torno O, García S, Prat M, Santamaría B. 1996. Enteroparasitosis del perro en un sector de Bahía Blanca, Argentina. Parasitol al Día 20: 144-146.

35. Tortolero L, Cazorla D, Morales $O$, Acosta ME. 2008. Prevalencia de enteroparásitos en perros domiciliarios de la Ciudad de la Vela, estado Falcón, Venezuela. Rev Cient 18: 312-319.

36. Trillo-Altamirano MP, Carrasco AJ, Cabrera R. 2003. Prevalencia de helmintos enteroparásitos zoonóticos y factores asociados en Canis familiaris en una zona urbana de la ciudad de Ica, Perú. Parasitol Latinoam 58: 136-141. doi: 10.4067/S0717-77122003000300009

37. Vásquez LR, Campo VH, Vergara D, Rivera O, Cordero H, Dueñas J. 2004. Prevalencia de Toxocara canis y otros parásitos intestinales en caninos en la Ciudad de Popayán, México. Rev Fac Cienc Salud Univ del Cauca 7: 1-15.

38. Vilca F, Anccasi M. 2013. Enteroparásitos en perros (Canis familiares) y gatos (Felis catus) de la provincia de Puno. Rev Inv Altoandinas 15: 117-122. 